Recibido:

8-VII-2021

Aceptado:

9-VIII-2021

Publicado en línea: 13-VIII-2021
Dificultades en el tratamiento del liquen plano oral en paciente sistémicamente comprometido: reporte de caso y revisión de literatura

\section{Difficulties in the Treatment of Oral Lichen Planus in a Systemically Compromised Patient: Case Report and Review of the Literature}

Yadira V. Boza Oreamuno DDS, MScㄹ Fiorella Mora Abarca²

1. Profesora asociada, Departamento de Ciencias Diagnósticas y Quirúrgicas, Facultad de Odontología, Universidad de Costa Rica, Costa Rica. https://orcid.org/0000-0002-0367-8664

2. Estudiante de quinto año de Licenciatura en Odontología, Facultad de Odontología, Universidad de Costa Rica, Costa Rica. https://orcid.org/0000-0002-3887-7129

Autor para correspondencia: Dra. Yadira V. Boza Oreamuno - yadira.boza@ucr.ac.cr

RESUMEN: El liquen plano oral (LPO) es una enfermedad crónica inflamatoria e inmunomediada, que se presenta como lesiones orales bilaterales, simétricas con tres presentaciones clínicas principales: reticular, atrófico y erosivo. Se caracteriza clínicamente por dolor, sensación de ardor y malestar para las personas que lo padecen. Actualmente LPO se clasifica como un desorden oral potencialmente maligno. Se ha relacionado con numerosas connotaciones sistémicas como diabetes mellitus, hipertensión, síndrome metabólico, enfermedades de la tiroides, dolencias psicosomáticas, enfermedad hepática crónica y enfermedades gastrointestinales. El diagnóstico se realiza mediante exámenes clínicos e histológicos. Es una enfermedad no curable, los tratamientos se aplican buscando la reducción o desaparición del dolor asociado, así como mejorar el aspecto clínico de las lesiones de carácter erosivoulcerativo. El objetivo de este trabajo fue presentar por medio de un caso clínico las dificultades en el tratamiento de LPO en un paciente sistémicamente comprometido, resaltando la valoración integral del mismo, terapias farmacológicas fallidas, hasta disminuir la sintomatología y mejorar la calidad de vida del paciente.

PALABRAS CLAVE: Liquen plano; Diabetes mellitus; Dislipidemia; Hipertensión arterial; Tratamiento. 
ABSTRACT: Oral lichen planus (OLP) is a chronic inflammatory and immune-mediated disease that presents as bilateral, symmetrical oral lesions with three main clinical presentations: reticular, atrophic, and erosive. It is clinically characterized by pain, burning sensation, and discomfort for sufferers. Currently OLP is classified as a potentially malignant oral disorder. It has been associated with numerous systemic connotations such as diabetes mellitus, hypertension, metabolic syndrome, thyroid diseases, psychosomatic ailments, chronic liver disease, and gastrointestinal diseases. Diagnosis is made by clinical and histological examinations. It is a noncurable disease, the treatments are applied seeking the reduction or disappearance of the associated pain, as well as improving the clinical appearance of erosive-ulcerative lesions. The objective of this work was to present through a clinical case the difficulties in the treatment of OLP in a systemically compromised patient, highlighting the comprehensive assessment of the same, failed pharmacological therapies, until the symptomatology diminished and the patient's quality of life improved.

KEY WORDS: Lichen planus; Mellitus diabetes; Dyslipidemia; Arterial hypertension; Treatment.

\section{INTRODUCCIÓN}

El liquen plano (LP) es una enfermedad mucocutánea crónica inflamatoria e inmunomediada (1). Para el liquen plano oral (LPO) se ha informado una prevalencia combinada global del 1,01\%, con una marcada diferencia geográfica, siendo más alta en Europa y más baja en la India, aumentando de forma significativa y progresiva a partir de los 40 años (2), mayor entre las mujeres en comparación con los hombres (3).

El 20\% de los casos de LPO se presentan con manifestaciones cutáneas (4), que en su mayoría son autolimitadas y provocan prurito, las lesiones orales suelen ser crónicas, alternando entre períodos de latencia y exacerbación (5). En la cavidad bucal, generalmente la mucosa oral, la lengua y las encías son los sitios más afectados (6), hay tres presentaciones clínicas principales: reticular, atrófico y erosivo (7). Se presenta clásicamente como una afección bucal bilateral (8), incluye placas hiperqueratósicas blancas que dan una apariencia de encaje (9).

En los pacientes con LPO las quejas varían desde una sensación asintomática de aspereza de la mucosa hasta una sensación de ardor y dolor intenso, que puede determinar dificultades para comer, hablar y tragar (10). Estos síntomas clínicos pueden hacer que los pacientes sean propensos al desarrollo de estrés, ansiedad y depresión, lo que empeora el cuadro (11). Además, el LP se ha relacionado con numerosas connotaciones sistémicas como diabetes mellitus (DM), hipertensión, síndrome metabólico, enfermedades de la tiroides, dolencias psicosomáticas, enfermedad hepática crónica, enfermedades gastrointestinales y susceptibilidad genética al cáncer $(4,12,13)$.

Actualmente LPO se clasifica como un desorden oral potencialmente maligno $(14,15)$. 
El diagnóstico se realiza mediante exámenes clínicos e histológicos. Los tratamientos se aplican buscando la reducción o desaparición del dolor asociado, así como la mejora en el aspecto clínico de las lesiones de carácter erosivo-ulcerativo (16), controlar con ellos las fases agudas o los picos de exacerbación (1).

El objetivo de este trabajo es presentar por medio de un caso clínico las dificultades en el tratamiento de LPO en un paciente sistémicamente comprometido.

\section{REPORTE DE CASO}

Paciente masculino de 68 años de edad, vecino del Área Metropolitana, casado, pensionado. Se presenta a la Facultad de Odontología (FO) de la Universidad de Costa Rica (UCR) con la queja principal "tengo roto y me duele la lengua y parte interna de la mejilla". En el interrogatorio directo reporta antecedentes sistémicos: hipertensión, pre diabetes y una hernia hiatal, tratamiento actual con Irbesartan 150mg (2 en la mañana), Atenolol 50mg (1 al día), Amlodipina 5mg (1 al día), Metformina clorhidrato 500mg (1 al día), suplementos naturales como cápsulas de moringa (1 al día), salvado de avena. Consumió bebidas alcohólicas por más de 50 años, sin embargo, tiene dos años sin consumir licor, no consume tabaco ni otras drogas. Refiere que el dolor bucal fue apareciendo progresivamente en los últimos 8 meses, asociados a áreas rotas que le molestan para hablar y tragar los alimentos, los cuales para ingerirlos tienen que ser en forma de papilla, ya que no soporta alimentos duros, actualmente escala visual análoga (EVA) 9 y siente que ha bajado de peso porque la ropa le queda floja. Además, de estar muy desanimado por lo mal que se siente, no quiere ni compartir con la familia.

Al realizar el examen clínico se observó dorso de lengua saburral, de forma bilateral lesiones tipo placas blancas en forma de redes que no se remueven con gasa, estrías de Wickham, en combinación con áreas atróficas-eritematosas y erosivas-ulcerativas en ambas mucosas yugales, piso de boca, ventral de lengua y encía por vestibular (Figura 1), el paciente refiere mucho dolor bucal. Signo de Nicolsky negativo.

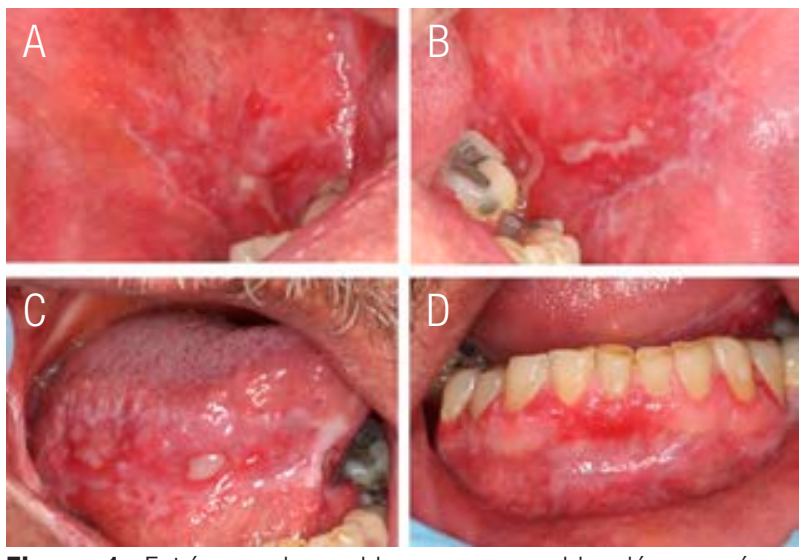

Figura 1. Estrías y placas blancas en combinación con áreas atróficas-eritematosas y erosivas-ulcerativas en mucosa yugal derecha $(A)$ e izquierda $(B)$, borde lateral lengua $(C)$ y zona erosiva en encía (D).

A nivel de cuello no se encontraron ganglios palpables. No se observaron alteraciones de contorno facial, ni en las uñas, ni en piel expuesta. Sin embargo, al consultarle por lesiones en otras partes del cuerpo menciona que lleva mucho tiempo (no precisa cuánto) de tener cuadros de "alergia" en el cuerpo, son "ronchas que pican y molestan", en piel de ambas piernas presentaba pápulas y placas violáceas ulceradas y con costra, refiere prurito (Figura 2). 


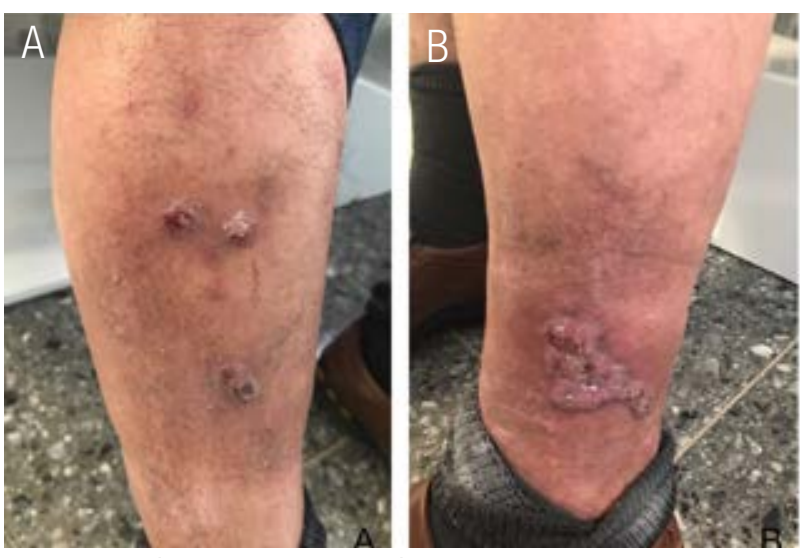

Figura 2. Pápulas y placas violáceas con reticulado blanquecino en combinación con úlceras y costras en superficie de la cara posterior de la pierna derecha (A) y la pierna izquierda (B). Obsérvese las zonas de pigmentación residual color gris-azuloso alrededor de las lesiones.

Bajo el diagnóstico clínico de LP erosivo y los diagnósticos diferenciales: pénfigo vulgar y penfigoide de las membranas mucosas. Se realizó biopsia incisional en mucosa yugal derecha. El estudio histopatológico mostró hiperqueratosis y acantosis, por debajo del epitelio de revestimiento se observó infiltrado inflamatorio crónico de predominio linfocitario denso en banda, que llega hasta el epitelio escamoso con degeneración vacuolar de la capa basal y presencia de cuerpos de Civatte asociados a los focos de destrucción de la capa basal (Figura 3), con diagnóstico de LP.

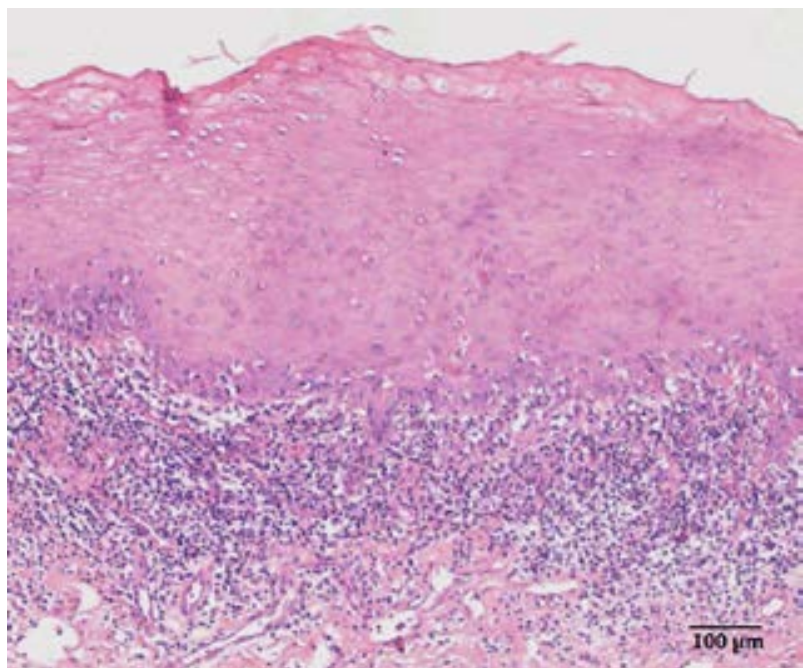

Figura 3. Hiperqueratosis, acantosis, degeneración hidrópica de la capa basal, infiltrado linfocitario en banda y lesión de interfase $(100 \mu \mathrm{m}, \mathrm{H} \& \mathrm{E})$.
Se le explicó al paciente su enfermedad y la importancia de un manejo integral para su caso, que incluye exámenes para valorar su estado sistémico, tratamiento y seguimiento en odontología y dermatología. El paciente solicitó ser tratado tanto en la F0 de la UCR y por medio de la Caja Costarricense del Seguro Social (CCSS), ya que no puede costear los estudios, un dermatólogo y el tratamiento de manera privada. Por lo que fue referido a la CCSS para la realización de hemograma completo, prueba de hepatitis C, función renal y hepática, niveles de glucemia, colesterol, perfil lipídico, hierro, vitamina B12 y ácido fólico, así como, al servicio de Dermatología, para una revaloración del caso y medicarlo. Se le dieron instrucciones para evitar traumas de la mucosa bucal, continuar con la dieta blanda, evitar: condimentos, gomas de mascar, jugos de frutas ácidos o bebidas carbonatadas, tener mucha hidratación y evitar el estrés. Se le envió pasta dental Oddent $\AA$ Desensibilizante y 0 ddent $\AA$ ácido hialurónico en spray 3 veces al día.

Los resultados de los exámenes de laboratorio fueron dentro de límites normales, excepto elevado colesterol, las lipoproteínas de baja densidad (LDL) $130.2 \mathrm{mg} / \mathrm{dL}$ (normal<100), le prescribieron lovastatina 20mg una por noche. En dermatología para uso oral se iniciaron con dapsona $100 \mathrm{mg}$ y fexofenadina hidrocloruro $120 \mathrm{mg}$ y para las lesiones cutáneas betametasona base al 0,1\%, luego de 22 días con el tratamiento los síntomas orales mejoraron un poco, sin embargo, el paciente presentó descamación en piel siendo muy marcada en las palmas de las manos, lo que se atribuyó a una dermatitis exfoliativa, reacción adversa ocasionada por la dapsona (Figura 4), el dermatólogo le cambió la medicación por azatioprina $50 \mathrm{mg}$ y prednisolona $50 \mathrm{mg}$ diarios por día por semana, se le adicionó un enjuague bucal diario por la noche con $5 \mathrm{ml}$ de nistatina 100.000 $\mathrm{Ul}$ en suspensión oral $/ \mathrm{ml}$ para prevenir sobre infección micótica. Mejoró significativamente, las lesiones en las piernas y las manos (Figura 5), 
pero lamentablemente, el paciente a la semana empezó a experimentar mucho dolor estomacal, náuseas y vómitos, por lo que se sospecha de la nueva medicación, el dermatólogo le suspende la Azatioprina, continuó con la Prednisolona bajando la dosis de forma gradual hasta $5 \mathrm{mg}$ como dosis de mantenimiento.

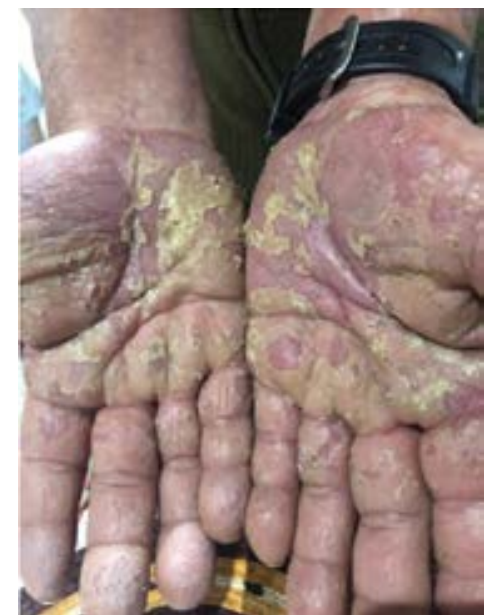

Figura 4. Palmas de ambas manos con descamación asociado al uso de dapsona.

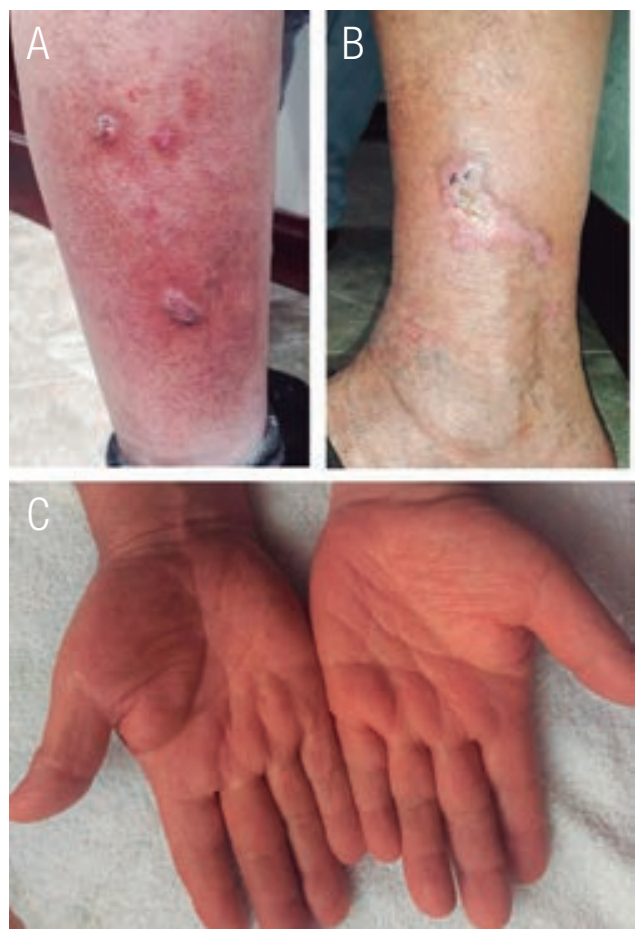

Figura 5. Mejoría a las 2 semanas en las lesiones cutáneas correspondientes a las piernas con el tratamiento sistémico inicial (A). Ambas palmas de las manos 2 semanas después de suspender la dapsona (B).
Se le ha realizado seguimiento durante un año y medio, manteniendo un control para un buen estado periodontal y dental, reforzando la higiene oral y la importancia de una alimentación balanceada, logrando niveles de colesterol normales. El paciente refiere sentirse bien, continuando con los cuidados y la medicación indicados, ha presentado algunas áreas ulceradas en cavidad bucal por trauma en mucosa yugal pero resuelven utilizando Oddent $\circledast$ ácido hialurónico en gel (Figura 6).
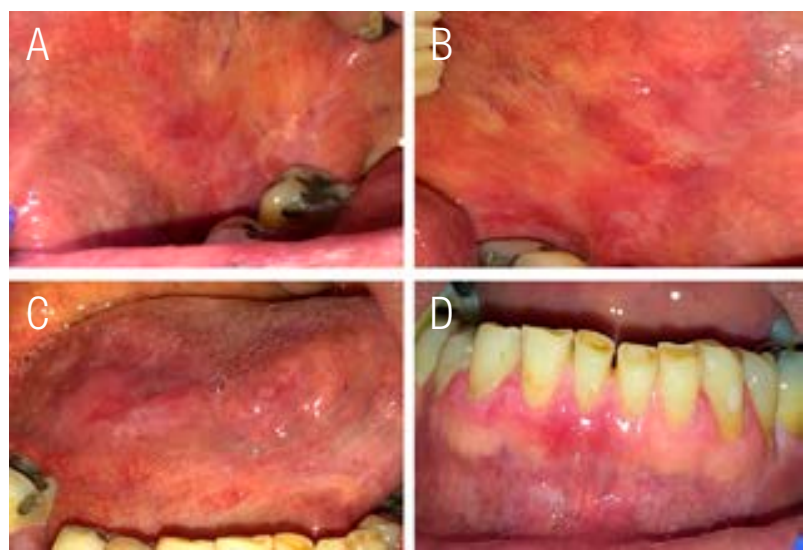

Figura 6. Disminución de las lesiones erosivas-ulcerativas y atróficas-eritematosas con permanencia de algunas lesiones reticulares en mucosa yugal derecha $(A)$ e izquierda (B), borde lateral derecho de lengua (C) y encía (D), en control a los 3 meses desde el diagnóstico.

El paciente dio por escrito su consentimiento libre e informado para la publicación de su caso.

\section{DISCUSIÓN}

El caso reportado presenta características clínicas e histológicas coincidentes con la literatura, con la diferencia que se trata de un hombre y el LPO se ha reportado con predominio en mujeres (3). Así mismo, se trata de un paciente diabético e hipertenso con niveles elevados de LDL, lo que resulta importante, ya que los pacientes con LP tienen más probabilidades de desarrollar síndrome metabólico (12).

Se ha informado que el LP se asocia con dislipidemia, indicada por un aumento de los 
niveles séricos de triglicéridos, colesterol y LDL, y niveles reducidos de lipoproteínas de alta densidad (HDL) (13). El metaanálisis realizado por Mozaffari y cols. mostró una alta prevalencia de LPO en pacientes diabéticos, un 9,3\% de los pacientes con DM sufrían LPO en comparación con los pacientes del grupo control, aunque su asociación no fue significativa (17). El estudio realizado por Kumar y cols. (18) indica que un $4,5 \%$ de los pacientes hipertensos de su estudio tenían presencia de lesiones de LPO.

Se ha destacado la necesidad de una evaluación exhaustivayenfoques multidisciplinarios de esta enfermedad (6). Lo que en nuestro caso fue muy importante al someter al paciente a una minuciosa anamnesis y exploración para investigar posibles manifestaciones extraorales, la importancia de un manejo integral, con seguimiento nutricional, odontología y dermatología. Se recomienda en los pacientes con LPO iniciar con las medidas generales, una explicación concreta acerca de su padecimiento y su evolución, cómo mantener una buena higiene oral, evitar el consumo de alimentos y bebidas picantes, ácidos o muy condimentados, así como el consumo de tabaco y alcohol (19). Estas medidas de cuidado oral fueron de gran ayuda para la evolución de nuestro caso, sobre todo al ser un paciente sistémicamente comprometido.

Si bien, el LPO no es una enfermedad curable, se debe dar el tratamiento adecuado para ayudar a disminuir la sintomatología y mejorar la calidad de vida del paciente (20). Para ello, es importante considerar la cronicidad de la enfermedad, la presencia de lesiones en localizaciones extraorales, el historial médico del paciente, la comorbilidad y la condición de la cavidad oral (21). Sin embargo, en nuestro caso otro aspecto clave fue la parte económica y de ahí que el tratamiento fuera dado por medio de la seguridad social, lo cual, no podíamos controlar.
Los corticosteroides se han utilizado por vía tópica como medicamentos de primera línea, generalmente se reservan sistémicamente para LPO con presentación erosiva generalizada 0 exacerbación (22). García-Pola y cols. (23), en su revisión sistemática realizada en el 2017, destaca para tratar el LPO en sus formas clínicas atrófica y erosiva, como primer abordaje los glucocorticoides de mayor o menor potencia de aplicación tópica, en segundo lugar, los fármacos inhibidores de la calcineurina como primera opción el tacrolimús tópico al $0,1 \%$ y para aquellas lesiones persistentes de LPO se indica administración por vía sistémica de prednisona calculándolo de forma individualizada para cada paciente. En ausencia de respuesta con los corticosteroides, se ha informado que los retinoides pueden ser útiles, dapsona 0 ácido hialurónico (24).

Sin embargo, en nuestro caso el tratamiento inicialfuecondapsona. Sehareportadoquealrededor de un $1,5 \%$ de los pacientes que toman dapsona desarrollan un síndrome de hipersensibilidad, que se manifiesta de 2 a 6 semanas tras el inicio del tratamiento (25), este se caracterizada por algunas o todas de las siguientes manifestaciones: dermatitis exfoliativa o eritrodermia y/u otras erupciones cutáneas, linfadenopatía generalizada, fiebre, hepatitis y hepatoesplenomegalia (26). La dermatitis exfoliativa es un trastorno inflamatorio caracterizado por eritema con diversos grados y tipos de descamación, una de sus causas más comunes son las reacciones a medicamento (27). La suspensión del fármaco sospechoso y la mejoría de la eritrodermia son los principales criterios para confirmar el diagnóstico (28). Lo antes descrito coincide con nuestro caso reportado, donde el efecto adverso del medicamento fue mayor que la mejoría en el paciente.

Luego se continuó con azatioprina y predinisolona. La azatioprina tiene un papel 
interesante como terapia ahorradora de esteroides en el LP (29). Lamentablemente para el paciente, en nuestro caso las reacciones de dolor estomacal, náuseas y vómitos llevaron a la suspensión de la azatioprina, quedando medicado solo con la predinisolona, logrando eso sí disminuir las lesiones y la sintomatología.

Se debe considerar que los corticosteroides presentan efectos secundarios, que tienden a ser observados en un tratamiento sistémico prolongado, tales como síndrome de Cushing, hipertensión, diabetes, úlceras gástricas, inmunosupresión, infecciones fúngicas, etc. (1), de ahí la importancia que se administren de manera correcta bajando la dosis gradualmente. El uso de corticosteroides tópicos en la administración tópica puede tratar cualquier lesión menor residual (16).

Existe la necesidad de controlar a los pacientes con LPO durante un período prolongado, ya que algunos casos pueden sufrir transformación maligna (30). Revisiones sistemáticas y metanálisis han estimado las proporciones de transformación maligna de LPO en un rango de 0.44-2.28\% $(14,31)$. Estudios sugieren que la forma erosiva, el género femenino y la localización en lengua deben considerarse factores de riesgo para la transformación del LPO (32). Debemos destacar que en nuestro caso no observamos malignización de las lesiones, además, el paciente ya no tomaba bebidas alcohólicas y no fumaba, siendo el tabaco y el alcohol los principales factores de riesgo para carcinoma oral de células escamosas $(33,34)$.

Así mismo, se ha informado la importancia de la medición de la calidad de vida relacionada con la salud oral en pacientes con LPO, no solo como parte de la evaluación de los pacientes, sino para modular el tratamiento administrado en función de la respuesta (20), que en este caso el paciente no solo sufrió por la sintomatología de su enfermedad sino también por los tratamientos recibidos. Se ha reportado la necesidad de mejorar el conocimiento del LPO entre dentistas y dermatólogos a través de la educación continua (2), con lo que coincidimos y quedó en evidencia con este reporte de caso.

\section{CONCLUSIONES}

Es necesario valorar y estudiar de manera integral a un paciente con LPO, interrogar sobre la ingesta de fármacos y lesiones en otras partes del cuerpo, realizar una exploración física en busca de lesiones, apoyarse con exámenes de laboratorio y biopsia; además, de saber identificar la presentación clínica del LPO y su manejo terapeútico adecuado.

Por otra parte, es importante entender que se pueden presentar terapias farmacológicas fallidas que van a repercutir directamente en la calidad de vida de los pacientes, tanto en la parte física como aspectos sociales y psicológicos, por ello la importancia de un manejo multidisciplinario del caso para tratar a tiempo y de manera adecuada la sintomatología, además de hacer énfasis en la importancia de revisiones periódicas.

\section{CONFLICTO DE INTERESES}

Ninguno declarado.

\section{DECLARACIÓN DE CONTRIBUCIÓN DEL AUTOR}

Conceptualización y diseño: YBO, FMA.

Revisión de literatura: YBO, FMA.

Metodología y validación: YBO, FMA.

Análisis formal: YBO, FMA.

Investigación y recopilación de datos: YBO, FMA. Recursos: no aplica.

Análisis e interpretación de datos: YBO, FMA.

Redacción-preparación del borrador original: YBO, FMA.

Escritura-revisión y edición: YBO, FMA.

Supervisión: YBO.

Administración de proyectos: no aplica.

Adquisición de fondos: no aplica. 


\section{REFERENCIAS}

1. Mutafchieva M.Z., Draganova-Filipova M.N., Zagorchev P.I., Tomov G.T. Oral Lichen Planus - Known and Unknown: a Review. Folia Med (Plovdiv). 2018; 60 (4): 528-35.

2. González-Moles M.Á., Warnakulasuriya S., González-Ruiz I., González-Ruiz L., Ayén Á., Lenouvel D., et al. Worldwide prevalence of oral lichen planus: A systematic review and meta-analysis. Oral Dis. 2021; 27 (4): 813-28.

3. Li C., Tang X., Zheng X., Ge S., Wen H., Lin X., et al. Global Prevalence and Incidence Estimates of Oral Lichen Planus: A Systematic Review and Meta-Analysis. JAMA Dermatology. 2020; 156 (2): 172-81.

4. Hasan S., Ahmed S., Kiran R., Panigrahi R., Thachil J.M., Saeed S. Oral lichen planus and associated comorbidities: An approach to holistic health. J Fam Med Prim Care [Internet]. 2019; 8: 3504-17. Available from: $\quad$ http://www.jfmpc.com/article. asp? issn $=2249-4863$; year $=2017$; volume $=6$; issue $=1$; spage $=169$; epage $=170$; aulast $=$ Faizi

5. Alrashdan M.S., Cirillo N., McCullough M. Oral lichen planus: a literature review and update. Arch Dermatol Res. 2016; 308 (8): 539-51.

6. Cassol-Spanemberg J., Blanco-carrión A., Rivera-campillo M.E.R. Cutaneous, genital and oral lichen planus : A descriptive study of 274 patients. Med Oral Patol Oral Cir Bucal. 2019; 24 (1): e1-7.

7. Müller S. Oral Manifestations of Dermatologic Disease: A Focus on Lichenoid Lesions. Head Neck Pathol. 2011; 5 (1): 36-40.

8. Holmstrup P., Plemons J., Meyle J. Non-plaque-induced gingival diseases. J Clin Periodontol. 2018; 45: S28-43.
9. Cheng Y.S.L., Gould A., Kurago Z., Fantasia J., Muller S. Diagnosis of oral lichen planus: a position paper of the American Academy of Oral and Maxillofacial Pathology. Oral Surg Oral Med Oral Pathol Oral Radiol [Internet]. 2016; 122 (3): 1-23. Available from: http:// dx.doi.org/10.1016/j.oooo.2016.05.004

10. Parlatescu I., Tovaru M., Nicolae C.L., Sfeatcu R., Didilescu A.C. Oral health-related quality of life in different clinical forms of oral lichen planus. Clin Oral Investig. 2020; 24 (1): 301-8.

11. Adamo D., Cascone M., Celentano A., Ruoppo E., Leuci S., Aria M., et al. Psychological profiles in patients with symptomatic reticular forms of oral lichen planus: A prospective cohort study. J Oral Pathol Med . 2017; 46 (9): 810-7.

12. Ying J., Xiang W., Qiu Y., Zeng X. Risk of metabolic syndrome in patients with lichen planus: A systematic review and meta-analysis. PLoS One [Internet]. 2020; 15 (8): 1-13. Available from: http://dx.doi. org/10.1371/journal.pone.0238005

13. Panchal F., Ray S., Munshi R., Bhalerao S., Nayak C. Alterations in lipid metabolism and antioxidant status in lichen planus. Indian J Dermatol. 2015; 60 (5): 439-44.

14. González-Moles M.Á., Ruiz-Ávila I., González-Ruiz L., Ayén Á., Gil-Montoya J.A., Ramos-García P. Malignant transformation risk of oral lichen planus: A systematic review and comprehensive meta-analysis. Oral Oncol [Internet]. 2019; 96: 121-30. Available from: https://doi.org/10.1016/j. oraloncology.2019.07.012

15. Warnakulasuriya S. Oral potentially malignant disorders: A comprehensive review on clinical aspects and management. Oral Oncol [Internet]. 2020; 102 (December 2019): 104550. Available from: https://doi. org/10.1016/j.oraloncology.2019.104550 
16. Rotaru D.I., Sofineti D., Bolboacă S.D., Bulboacă A.E. Diagnostic Criteria of oral lichen planus: A narrative review. Acta Clin Croat. 2020; 59 (3): 513-22.

17. Mozaffari H.R., Sharifi R., Sadeghi M. Prevalence of oral lichen planus in diabetes mellitus: A meta-analysis study. Acta Inform Medica. 2016; 24 (6): 390-3.

18. Kumar P., Mastan K.M.K., Chowdhary R., Shanmugam K. Oral manifestations in hypertensive patients: A clinical study. J Oral Maxillofac Pathol. 2012; 16 (2): 215-21.

19. Aghbari S.M.H., Abushouk A.I., Attia A., Elmaraezy A., Menshawy A., Ahmed M.S., et al. Malignant transformation of oral lichen planus and oral lichenoid lesions: A meta-analysis of 20095 patient data. Oral Oncol [Internet]. 2017; 68: 92-102. Available from: http://dx.doi.org/10.1016/j. oraloncology.2017.03.012

20. Yuwanati M., Gondivkar S., Sarode S.C., Gadbail A., Sarode G.S., Patil S., et al. Impact of Oral Lichen Planus on Oral Health-Related Quality of Life: A Systematic Review and Meta-Analysis. Clin Pract. 2021; 11 (2): 272-86.

21. Florek A.G., Wang C.J., Armstrong A.W. Treatment preferences and treatment satisfaction among psoriasis patients: a systematic review [Internet]. Vol. 310, Archives of Dermatological Research. Springer Berlin Heidelberg; 2018. 271-319 p. Available from: http://dx.doi.org/10.1007/ s00403-018-1808-x

22. Nosratzehi T. Oral lichen planus: An overview of potential risk factors, biomarkers and treatments. Asian Pacific J Cancer Prev. 2018; 19 (5):1161-7.
23. García-Pola M.J., González-Álvarez L., Garcia-Martin J.M. Tratamiento del liquen plano oral. Revisión sistemática y protocolo de actuación. Med Clin (Barc) [Internet]. 2017; 149 (8): 351-62. Available from: http:// dx.doi.org/10.1016/j.medcli.2017.06.024

24. Al-Hashimi I., Schifter M., Lockhart P.B., Wray D., Brennan M., Migliorati C.A., et al. Oral lichen planus and oral lichenoid lesions: diagnostic and therapeutic considerations. Oral Surgery, Oral Med Oral Pathol Oral Radiol Endodontology. 2007; 103 (SUPPL.): S25.e1-S25.e12.

25. Ordieres Ortega L., Temiño Frade C., Demelo-Rodríguez P. Late onset dapsone hypersensitivity syndrome. Med Clin (Barc). 2019; 153 (5): e23-4.

26. Agrawal S., Agarwalla A. Dapsone Hypersensitivity Syndrome: A ClinicoEpidemiological Review. J Dermatol. 2005; 32: 883-9.

27. Salami T.A.T., Enahoro Oziegbe O., Omeife H. Exfoliative dermatitis: Patterns of clinical presentation in a tropical rural and suburban dermatology practice in Nigeria. Int $\mathrm{J}$ Dermatol. 2012; 51 (9): 1086-9.

28. Miyashiro D., Sanches J.A. Erythroderma: a prospective study of 309 patients followed for 12 years in a tertiary center. Sci Rep. 2020; 10 (1): 1-13.

29. Husein-ElAhmed H., Gieler U., Steinhoff M. Lichen planus: a comprehensive evidencebased analysis of medical treatment. J Eur Acad Dermatology Venereol. 2019; 33 (10): 1847-62.

30. Tsushima F., Sakurai J., Uesugi A., Oikawa Y., Ohsako T., Mochizuki Y., et al. Malignant transformation of oral lichen planus: a 
retrospective study of 565 Japanese patients. BMC Oral Health [Internet]. 2021; 21 (1): 1-9. Available from: https://doi.org/10.1186/ s12903-021-01652-7

31. González-Moles M.Á., Ramos-García P. W.S. An appraisal of highest quality studies reporting malignant transformation of oral lichen planus based on a systematic review. Oral Dis. 2020; Epub ahead.
32. Giuliani M., Troiano G., Cordaro M., Corsalini M., Gioco G., Lo Muzio L., et al. Rate of malignant transformation of oral lichen planus: Asystematic review. Oral Dis. 2019; 25 (3): 693-709.

33. Ernani V., Saba N.F. Oral cavity cancer: Risk factors, pathology, and management. Oncol. 2015; 89 (4): 187-95.

34. Wong T.S.C., Wiesenfeld D. Oral Cancer. Aust Dent J. 2018; 63 (1): S91-9. 